\title{
Carnets
}

Revue électronique d'études françaises de l'APEF

Deuxième série - 17 | 2019

Lîlle : prisme de la connaissance ou reconnaissance du monde

\section{L'île en tant qu'elle est un trou}

\section{Lionel Rebout}

\section{OpenEdition}

Journals

Édition électronique

URL : http://journals.openedition.org/carnets/10313

DOI : 10.4000/carnets.10313

ISSN : 1646-7698

Éditeur

APEF

Référence électronique

Lionel Rebout, "L'île en tant qu'elle est un trou », Carnets [En ligne], Deuxième série - 17 | 2019, mis en ligne le 30 novembre 2019, consulté le 07 janvier 2020. URL : http://journals.openedition.org/carnets/ 10313 ; DOI : 10.4000/carnets. 10313

Ce document a été généré automatiquement le 7 janvier 2020.

\section{(c) (7) (9)}

Carnets est mis à disposition selon les termes de la licence Creative Commons - Atribution - Pas d'utilisation commerciale 4.0 International. 


\title{
L'île en tant qu'elle est un trou
}

\author{
Lionel Rebout
}

1 Dépassons l'incongruité du trou et de son impensé chronique pour dire en quoi le trou grandit ou altère l'insularité. Voilà une rare occasion d'appréhender le trou ${ }^{1}$ et de mieux le comprendre quand il est en relation avec l'espace insulaire. Quel dialogue entretiennent-ils entre eux ? Comment le trou participe-t-il à la vie insulaire, mais plus encore à son imaginaire ? Et en quoi devient-il nécessaire à la constitution de celui-ci ? Comment l'un permet-il de définir l'autre sous un angle anthropique? Le trou comme l'île ont en commun de se situer entre une présence et une absence. Alors qu'une île n'a pas toujours un ancrage bien fondé, sa géographie relève donc de l'incertitude, du flou parfois, ce qui explique les péripéties diplomatiques et juridiques entre États.

2 Dès l'émergence jusqu'à la disparition forcée de l'espace insulaire, le trou en est souvent la cause ou la conséquence. Si l'existence en propre de l'île en dépend ou en résulte, le trou se présente dans bien des positions différentes. Tantôt il encercle l'île ; la creuse ou la porte; l'engloutit comme une grande bouche; la traverse, la perce. Tantôt le trou reste la seule voie pour la survie. Comprenez qu'à sa disparition, le trou reste souvent la seule trace de l'île passée.

3 À d'autres moments de son existence, l'île présente une forte concentration de trous. Souvent elle reste le meilleur endroit pour cacher des trous et des expériences inavouables qui doivent demeurer invisibles car la définition de l'île se superpose à l'usage que l'on en fait. Une partie de l'insularité dépend du trou, lequel permet d'achever l'existence de l'île et même la prolonge. Il l'accompagne toute la vie durant, sans être un obstacle à celle-ci, ni même un ennemi invisible. À bien y compter, si le trou vient à faire du mal à l'île, cela se fera souvent par inadvertance et très rarement. Le trou permet une respiration à la manière d'un corps qui souffle l'air, les gaz et qui projette les eaux, irriguant la vie. Et pourquoi pas le soleil, sachant que l'inhospitalité de certaines îles se voit ainsi contrecarrée par diverses énergies salvatrices!

4 Il s'agira dans notre propos de décliner le trou sous des acceptions différentes afin de mettre en lumière la relation singulière entre le trou et l'île. La réalité insulaire est $\mathrm{si}$ vaste et ambivalente (Meistersheim, 1988: 108), tantôt concentration de l'espace continental, tantôt distorsion de celui-ci, que l'on peut avoir une approche différente, 
originale entre le tangible et le mythique, au point d'associer l'île au trou. L'insularité par sa singularité secoue les fondations de l'espace continental quand la réalité physique rejoint le politique, l'économique, l'anthropologique et le langage quand ce dernier nourrit le registre symbolique et la mythologie.

5 Alors qu'il existe environ 300000 îles dans le monde, quel lien peut-il exister entre l'insularité et le trou? Contrairement à ce que l'on penserait au premier abord, d'un point de vue conceptuel ou d'un point de vue géographique, l'île et le trou sont très proches en réalité. Dès l'Antiquité, au travers des poèmes homériques, l'espace insulaire et le trou sont réunis selon les épisodes, se superposant même ${ }^{2}$.

6 L'un et l'autre sont une hétérotopie au bord incertain parfois, au sens de Foucault (Foucault, 1984), lequel nous servira plus loin afin de décliner la relation entre l'espace insulaire et le trou. Plus encore, le premier comme le second sont un interrupteur du temps et de l'espace, facilitant le passage de la vie ou la freinant brutalement. À ce titre, l'île participe d'une pensée géographique aux accents poétiques et philosophiques en tant que signe face à des corps constitués et articulés, en tant que concrétion, vide, possibilité d'une vie désolée ou magnifiée par sa position et ce qu'elle permet, loin du monde. Grain de beauté, bien plus que fragment de continent, l'île touche au langage qu'elle ne peut laisser indifférent. Marquant la coupure à laquelle oblige le monde, elle permet le sentiment, la parole, par conséquent une pensée magique et onirique.

7 Envisager l'île en tant que trou est une porte d'entrée principale pour mieux comprendre ce que représente ce confetti. Par exemple au Québec, l'image de l'île de René-Levasseur vue du ciel est saisissante en raison du cercle d'eau parfait qui l'entoure. Une île est autant du plein que du vide ; autant une bouée de sauvetage qu'un trou perdu. Dire de l'île qu'elle est un trou est une façon de mieux la comprendre, la saisir dans l'océan de la pensée concrète, mythologique et politique. Réunion du faire et du penser, à la tangente du monde, dent de la mer qui déchiquète les bateaux, l'île est entendue comme un trou qui ne dit pas toujours son nom. Trou perdu, trou que l'on ne parvient pas à retrouver, trou que l'on ne parvient pas à nommer et que l'on cherche jusque sur le bout de sa langue. En face de Brest, flotte littéralement l'île Perdue à la limite de la surface atlantique, avec une altitude d'à peine trois mètres. D'où son nom approprié.

\section{Lîle faite enfermement et damnation}

8 En quoi l'insularité a à voir avec le trou ? On peut répondre à cette question par le biais du carcéral et du naufrage de Robinson sur une île. Être dans le trou, être en prison se rapproche d'une expérience de désolation, d'isolement proche de ce qu'a vécu Robinson sur son île (Rebout, 2019). Vivre sur une île, dans un espace limité, s'apparente à une vie tronquée telle que peut la vivre un détenu, un reclus, même si Robinson est enfermé dans un milieu ouvert, à la différence du second qui demeure dans une cellule fermée faite de béton. Parallèlement à la clôture, l'île comme la prison casse la routine, les codes, les habitudes activant une rupture sévère. Le récit de Robinson permet de comprendre en quoi une île est une prison, un piège, nourrissant de fait une analogie avec la vie carcérale. Selon ce point de vue, on ne retiendrait de l'île que le lieu d'une vie limite alors que le trou et l'île sont une occasion d'une vie unique et riche rendue possible grâce à la coupure du monde. D'ailleurs progressivement, la vie de Robinson s'ouvre mais difficilement, manière de reconquérir 
sa vie, jusqu'à la rencontre avec Vendredi mais plus encore après son départ de l'île. Il découvrira que son investissement financier a fructifié avec bonheur, sans lui, sans son travail directement. Libre à lui d'en jouir et de voyager. L'île de Robinson représente un trou dans sa vie qui finira par se remplir.

Nombreuses sont les îles qui ont servi de lieux de détention, de réclusion ${ }^{3}$. Plus proche de nous, le Mont-Saint-Michel a été un lieu de garnison à la fin du Moyen Âge et une prison du XV $v^{e}$ au XIXe siècle. Le bâti avait été adapté en conséquence.

10 Tous les continents semblent avoir besoin de leurs îles poubelles, lieu du recyclage social. Quand la géographie fonde le politique, l'île permet d'assigner socialement et politiquement l'individu à une place précise. Participant du territoire de la nation, l'île concrétise et rend bien réelle cette place de l'autre par l'attribution d'un lieu, en fonction de son caractère désigné. De leur côté, l'île de Sieck et l'île Longue furent un camp d'internement durant la Première Guerre mondiale. Plus récemment, les îles de Rab et de Pag en Croatie ont accueilli un camp de concentration au cours de la Seconde Guerre mondiale.

11 L'île d'Elbe, l'île de Chiloé, l'île d'Yeu, l'île Lipari ont été des lieux de bannissement, de déportation, conséquence d'une décision teintée de politique parfois. L'espace insulaire dans ce cadre est une banlieue, un lieu qui s'articule à un autre espace politique, valide face à ceux qui sont amputés sur un plan civique. De triste mémoire, il y a l'île de Sprogø au Danemark dans les années 1920, qui accueillait des femmes déficientes mentalement (mais si peu finalement) et moralement sous l'effet d'un mouvement hygiéniste. On y préconisait une rééducation par le travail selon la conception du Docteur Keller. Ce dernier a permis en 1929 une campagne de stérilisation. Ce centre fut fermé en 1961.

En tant que lieu de relégation sanitaire, autre type de mise à l'écart, une île devient le lieu idéal pour procéder à des quarantaines ${ }^{4}$, manière de filtrer et contrôler ce qui rentre: les biens et les personnes. La peste fait peur à juste titre. La quarantaine s'organise par la construction de lazarets, qui se confondent parfois avec des maladreries destinées à des individus contagieux, donc sales sur un plan politique et social. Ce type de sas d'entrée est l'une des conditions de l'existence d'une hétérotopie.

13 Autre trou et hétérotopie, le cimetière. Nombreuses sont les îles qui ont reçu des tombes et des corps. Il fallait écarter la violence de la mort, la force des esprits de retour chez les vivants. Ce type de choix a traversé les continents 5 . L'île engage une coupure entre le propre et le sale, le légal et l'illégal, la vie et la mort, peu importe les croyances. Tel un contrepoint, l'île permet donc de valider un espace politique viable pour les institutions et la cité. On écarte le danger autant qu'on se légitimise. De longue date, l'île constitue un voyage vers l'au-delà et des pratiques religieuses que seul ce type de territoire est en droit d'accepter. Sur les côtes celtiques, il n'est pas rare de déceler des dolmens et autres cairns ${ }^{6}$. Toute l'Europe semble concernée par ces pratiques funéraires.

14 Il faut dire que dans sa nature, par sa géologie et son isolement, l'île permet une œuvre au noir, un travail dans l'invisible, qui semble propice au monde carcéral et au sentiment religieux. Elle est le lieu des forces qui meurent, qui naissent, voire qui renaissent. Les dieux trouvent souvent dans l'île un lieu de naissance ou un lieu d'apprentissage (Vilatte, 1991). 

retrouve dans des terres entourées d'autres terres. Se détachent aussi les îles lacustres, fluviales, deltaïques, privées ou non et les îles artificielles. On a là un trait caractéristique de l'espace insulaire, mu par une force invisible, à la limite de la magie, susceptible de transformer la matière humaine, de relier la terre et le ciel. En cela, l'expérience insulaire doit être appréhendée à la manière d'un voyage esthétique dont cherchent à nous rendre compte la littérature et le cinéma ${ }^{7}$.

\section{Quand la langue se troue}

Au-delà de cette déclinaison à partir de la pensée foucaldienne de l'hétérotopie, il faut revenir à la description de l'espace insulaire et aux conditions d'émergence de celui-ci. Le trou est constitutif de l'île, laquelle creuse sa distance avec le continent, de la presqu'île qui largue les amarres lentement, aux échancrures, aux criques et aux calanques. Combien d'îles surgissent en passant sous silence les vides qui les constituent? Quand la volcanologie n'est pas loin, l'île semble être une boursoufflure de magma et de gaz, percée de grottes, de tunnels de lave. Aussi fragile que de la dentelle, un archipel peut se lire comme un réseau de tissu ouvragé.

Puis sous l'effet de l'usure du temps, des effondrements apparaissent. Nombre de Trou bleu ou de Blue Hole, de cénotes existent pour le plus grand bonheur des plongeurs et des touristes. Le trou sur une île est très souvent une hétérotopie à la limite d'une autre hétérotopie. Le fantasme ne va jamais assez loin dans ce langage avec l'imaginaire. Il se niche dans les recoins, les creux, les plis, les fissures de la terre. De ce point de vue, l'île reste une ligne fragile qui réunit l'eau et la terre, sous les mauvais auspices du vent. Confrontée à la limite, l'île est davantage exposée que le solide continent qui dispose de sa masse pour absorber les chocs et les tremblements.

18 Ainsi, le trou se révèle et trouve sa place par la toponymie insulaire. Chaque continent présente une liste diverse à la limite du vernaculaire et du folklorique ${ }^{8}$. Le long de la côte, on profite de geyser maritime appelé trou du souffleur sous l'effet de conduites naturelles qui renvoient le ressac de la mer. La Polynésie en compte plusieurs, La Réunion aussi. À partir de là, l'imaginaire reprend le relais : on parle de la narine de Neptune. Sans doute la terre doit-elle respirer, qui plus est avec grognements et soupirs. Sur l'île de Thassos, il y a le Drakotrypa, le trou du dragon. Un trou parmi d'autres qui s'habille selon les circonstances.

Modelant la terre et la roche, le trou produit les plages de sable dans les atolls, que l'esprit finit par nommer après coup. La ligne de démarcation avec la mer n'étant pas si solide, le trou exprime ainsi au travers de la concrétion une certaine porosité induite par la force des éléments. Passant d'une géographie physique à une géographie mentale, le trou permet cette élévation de l'esprit, du tangible vers le spirituel.

De son côté, l'histoire se construit à l'occasion dans quelques îles avec le trou. Par exemple sur l'île de Batz, un dragon a été terrassé en 525 au Trou des serpents par le moine Pol Aurélien. On a une légende similaire entendue dans l'île Callot. En Méditerranée, subsiste le trou des Pirates, un trou creusé par des corsaires en vue de cacher leur butin. Là on comprend qu'une île ne sert qu'à cela : faire un trou pour contenir tel un immense coffre-fort une fortune. La croix sur une carte est une sorte de 
sésame qui ouvre le trou à celui qui cherche et repousse l'adversité des océans, sans parler des animaux fabuleux qui rodent non loin.

21 Parfois, c'est l'île elle-même qui fait trou. Voyez l'île de la Pierre-Percée qui porte en elle le motif de son appellation. Il y a de quoi être interpellé : le lieu a inspiré un roman à Jules Sandeau en 1871. Autre exemple le Rocher Percé, une île canadienne dans le golfe du Saint-Laurent. La Norvège connaît une attraction semblable sur l'île de Torget. Un tunnel perce la montagne de granite. Voyez aussi l'arche naturelle dans l'île Ségal sur l'Atlantique. Et quand la nature n'est pas parfaite, l'homme invente une mythologie où le trou s'invite dans une île imaginaire : c'est le trou d'Orkey, pièce d'un jeu vidéo.

Si l'île est le lieu de la cachette, du refuge, de la croix sur une carte, elle désigne le trou perdu, représente le lieu du malfamé, de l'inhospitalier. Au Québec, pour dire que l'on va au bout du monde, on dit se rendre sur l'île Moukmouk, terre bien réelle sur le lac Duparquet. Par définition, un trou est mal identifié d'où le recours à des îles lointaines, infestées d'animaux féroces.

\section{Îles disparues, îles disparaissantes}

Sans parler des îles qui représentent un vrai paradis fiscal, un trou noir, qu'est-ce qu'une île engloutie sinon une île qui tombe dans un trou, encore plus en temps de guerre? Quand une île semble tenir sa valeur de son apparition, considérons sa perte, quitte à ce que la légende reprenne le dessus, telle l'Atlantide. $\mathrm{Au} \mathrm{xx}{ }^{\mathrm{e}}$ siècle, Elugelab a eu le triste sort d'être pulvérisée par une explosion atomique en 1952. Cet îlot a été le lieu d'essai de la première bombe à hydrogène dans le Pacifique. Un cratère de 1,6 km de diamètre se forma sur 50 mètres de profondeur. Ce trou servit deux fois par la suite. Puis, ce fut le tour de l'îlot voisin Teiter. Le Pacifique devient le théâtre dès l'année 1946 d'expériences que seuls les déserts ou les îles peuvent recevoir et contenir. On a recours à l'île envisagée comme un laboratoire, un espace insulaire qui peut tout et même l'horreur que l'on écarte ainsi du cœur de la cité.

Rappelons-nous que l'essentiel des 200 essais nucléaires français ont été déclenchés en Polynésie. Une belle preuve de déni de civilisation. L'Algérie tout d'abord, puis le lointain exotique, la meilleure assurance que la cité reste propre longtemps.

Plus proche de nos côtes, l'île de Cézembre a été une des premières îles où on a utilisé des bombes au napalm en 1944, une première en Europe, après l'île de Tinian dans le Pacifique. Les bombardements intensifs ont modifié le relief et la végétation à la suite. On a compté plus de 20000 bombes. S'en sont suivies deux grandes campagnes de déminage, a priori insuffisantes. On observe conséquemment plus de 2000 cratères. La guerre est souvent une affaire de trous face à d'autres trous.

Sur l'île de Borkum, dans la mer du Nord, laquelle interdisait la venue des touristes juifs, les Nazis ont développé en 1934 une fusée prototypique annonçant les V2. Plus tard, suite à de lourds bombardements anglais, leurs essais se sont poursuivis en 1942 sur l'île d'Usedom par von Braun, lequel connaissait déjà l'insularité puisqu'il avait été placé dans un internat par ses parents sur l'île de Spiekeroog. Plus tard, il finira sur le continent américain, aboutissement de son périple.

L'histoire nous montre que la guerre n'épargne aucun territoire. À ce titre, l'île n'est pas une terre mineure. Elle est parfois l'enjeu d'âpres combats et de renversements dans l'occupation car l'île reste un poste avancé lors de reconquêtes territoriales. 
Souvenons-nous des combats acharnés dans le Pacifique entre armée japonaise et américaine, d'île en île, d'archipel en archipel. Un engagement qui avait débuté par l'attaque surprise de Pearl Harbour, autre île. Une guerre qui s'est finie par les bombardements d'Hiroshima et de Nagasaki, sises sur deux grandes îles dans un Japon impérial et insulaire.

L'espace insulaire représente parfois un laboratoire au travers d'un espace seul capable de contenir l'expérience scientifique comme s'il fallait tenir à l'écart les dangers qui en découleraient. Le même besoin de mise à distance demeure lorsqu'il s'agit de faire de l'île une prison. Il faut cacher et probablement mythifier ce type de lieu, confondre le moyen et le but, le rendre concret dans sa perte. Dans les deux cas, l'isolement permet que le processus mental ou physico-chimique ne soit pas contrarié par un extérieur, source de pollution. L'espace insulaire permet ainsi la pureté. Il en est la garantie, un conservatoire d'espèces naturelles autorisant une reproduction authentique de la nature. Par exemple, les abeilles loin de toute contamination. L'île se fait alors sanctuaire, écrin d'une vie originale et primordiale.

\section{Des îles au statut incertain}

Voici quelques cas qui présentent des îles dont le statut est incertain en raison du droit ou de fondements géologiques assez peu ordinaires, quand la guerre rejoint la diplomatie et les relations internationales. J'expose ici rapidement plusieurs types de situations ou de statuts.

Qu'est-ce qu'une île traversée par une frontière sinon un no man's land, la marque d'un vide intersidéral dont le langage ne sort pas indemne ? Évidemment, le trou n'est pas loin. Chypre est un bon exemple car l'île est cisaillée entre plusieurs entités, entretenues par des forces internationales. On retrouve sur l'île de Malouine orientale, une zone appelée no man's land, en espagnol Tierra de nadie, du fait de l'aridité du secteur. Cet exemple nous ramène à l'inhospitalité de ces îles. Nuage de terre, piton rocheux, l'espace insulaire est quelquefois la matérialisation d'un vide, le lieu parfait pour des manœuvres militaires. On peut jouer à la guerre sans gêner les voisins.

Sans être un no man's land, il existe des territoires incertains dont la souveraineté n'est pas bien établie. Ce sont les micronations ${ }^{9}$. À côté de ce statut particulier, on retrouve cet autre curieux objet de droit Terra nullius, espace qui peut être habité mais qui reste sans maître ${ }^{10}$. Dans le prolongement, il reste encore les îles au nom flou. On use du terme anglais inchoate title. Mais l'incertitude territoriale ne tient pas qu'au droit. La nature géologique de l'espace insulaire entre en ligne de compte: son identité en dépend directement. Le statut ambigu d'une île correspond aux fantasmes et projections politiques d'autres États qui les convoitent (Fumey, 2002 et Fuligni, 1997). Se prêtant donc à des jeux sans importance, à la facétie qui n'est jamais loin, l'île Carnevalis montre que certains territoires n'ont guère de valeur écologique, diplomatique ou économique.

31 L'espace insulaire dit beaucoup de l'état des relations internationales. Si les îles font l'objet de bataille, en revanche d'autres îles sont traversées par des frontières sans heurt. Telle que l'île de l'écluse d'Apach sur la Moselle partagée inégalement entre le Luxembourg, l'Allemagne et la France. Si une île est inhabitée, cela rend quelquefois les relations internationales plus apaisées. 


\section{Faire son trou ou habiter une île}

d'y faire son trou, non pas pour assimiler l'île à un continent mais seulement pour exprimer cette faculté d'habiter. À travers les âges et les conquêtes humaines, il est fabuleux d'observer l'énergie, l'obstination des insulaires à faire leur trou, à rendre hospitalière une terre isolée, une terre ingrate sans les ressources naturelles que l'on retrouve en masse sur le continent. L'île Hierro aux Canaries est un parfait exemple d'indépendance énergétique grâce à l'hydroélectrique et l'éolien. Encore une affaire de trous qui va bien au-delà de sa matérialité. Les habitants expriment ici une volonté farouche d'habiter une terre, une volonté qui excède la terre, quand elle transcende l'humanité.

35 À travers le critère de l'habitabilité d'une île, on pourrait ainsi construire une hiérarchie et des seuils dans un classement, autorisant à dire ce qu'est une île, au-delà de la sempiternelle définition d'une terre entourée d'eau (Taglioni, 2006). Une île d'à peine $1 \mathrm{~km}^{2}$ laisserait suggérer une habitabilité insuffisante pour faire son trou. Faudrait-il encore ne pas être seul ! En deçà, l'île serait le théâtre d'une situation-limite impropre à la vie elle-même. Le caractère de l'habitable et donc de l'hospitalier semble pertinent afin que l'insulaire produise une subjectivité digne de ce nom et par là même crée une habitation selon la pensée de Heidegger. Ainsi les trous que l'on observerait sur les îles de petites superficies n'auraient que peu de valeurs, à peine seraient-ils mécaniques et circonstanciels. le moins une subjectivité construite de l'extérieur, selon un regard que l'on projetterait depuis le continent. Voici poindre l'île comme lieu des morts, lieu de bagnes et de prisons, lieu d'internement et de camps durant les guerres, lieu de monastères fermés aux non-initiés. Chaque île marque une césure entre une partie de la population et le quidam. Il faut être (dis)qualifié pour séjourner sur ce territoire. À chaque fois, le trou n'est pas loin, lequel est alors au service d'une pensée plus vaste. 
37 À d'autres moments, séjourner sur une île est l'expression d'un rêve, d'un séjour organisé pour un seul, tel le millionnaire qui s'offre une propriété isolée, hors du monde, un paradis sur terre, une tour d'ivoire. Ou un séjour organisé par un club de vacances ou pour une émission de télévision afin d'accentuer le sensationnel face au sensoriel. Le mythe nimbe alors l'espace insulaire dans ses plus beaux atours. On en conclurait que l'on est mieux ici, plus intensément rendu au monde sur une île que sur le continent. La vie se donne pour vivante, plus vraie que nature car le rapport au monde change, son esthétique aussi, au travers d'une rupture matérialisée par l'étendue d'eau, si mince soit-elle. «L'île est le lieu où tend à se réaliser le moi, en quête de ses propres origines, à la poursuite de ses propres rêves » (Trabelsi, $2005:$ 7). L'île est l'opérateur d'une subjectivité construite sur une esthétique nouvelle de l'espace immédiat.

Le trou et l'île permettent de mêler le fond et la forme, le signifiant et le signifié, ce qui est rare en géographie. On en use par facilité et volonté de dire davantage qu'un simple mot. Joignant implacablement l'acte à la parole, l'île sert à reléguer des populations indésirables autant qu'elle produit un havre de paix. D'un côté, l'île est un enfer, de l'autre elle est un paradis. Parfois, on passe de l'un à l'autre en une fraction de seconde. Comment dépasser ces deux faces insulaires?

La réponse tient dans l'habiter et la construction d'un je qui se plait à ressentir et à se projeter. L'espace insulaire garantit une existence autre dans un environnement différent de celui du continent. Source d'un sensorium original, habiter sur une île participe d'un imaginaire spécifique. Si une maison nous habite, l'insularité renouvelle l'habitation et donne une place à la rupture esthétique qu'implique un séjour sur une île en tant qu'elle est une hétérotopie.

Objet d'une construction intellectuelle et mentale qui autorise une rupture esthétique, l'île est une porte d'entrée dans un autre monde car elle produit un autre point de vue, marqué par une frontière maritime bien visible. «L'île ne se conçoit pas sans la société qui l'habite. Une vraie île est une île habitée » (Péron, 1993:160). Cela corrobore l'idée de Sloterdijk qui dit qu'habiter relève d'un sentiment de soi dans l'espace. Habiter est une fonction et non pas une condition, en définitive un socle fondateur de tous les comportements, un trait fondamental de la condition humaine. L'homme dans son être même est un habitant. L'île est probablement le lieu où s'exprime davantage la question de l'habitation car faire son trou est la façon vulgaire de dire habiter au sens de Heidegger. Reste à dire ce que l'île permet ou non d'être et donc d'habiter. On aura compris que l'île procure une expérience esthétique particulière, à la faveur d'un voyage inédit. Tout sera inégalé désormais en raison de la coupure avec le temps précédent. En cela, l'île représente une épreuve du monde, dans le temps et dans l'espace. Loin d'être enfermé, un îlien peut se sentir libre sur son île car profondément enraciné et ressentira une forme de quiétude, termes synonymes pour le philosophe allemand (Heidegger, 1958).

\section{Circonscrire l'espace insulaire}

41 Comment comprendre ce qu'écrit Bonnemaison (1986) dans La dernière île? "Les peuples insulaires sont habités par le sentiment de la rupture.» Vivre sur une île c'est vivre l'île, vivre la rupture, se préparer à la rupture, la vouloir et en jouir. Mais pourquoi? Car il s'agit de disparaître en dernier lieu. En tant qu'hétérotopie, l'île 
permet de disparaître et c'est sa principale fonction, même si l'espace insulaire reste un marqueur, un point et un signe dans une pensée et un imaginaire construit dans l'espace (Nora, 2003). Si le trou coexiste avec l'espace insulaire dans sa trivialité, sa matérialité la plus pure, il participe d'un imaginaire, devenant l'élément d'une grammaire, probablement un opérateur. Il faut comprendre qu'il y a une gradation de la main qui perce la matière vers l'esprit qui habite une terre, peu importe le registre.

L'imaginaire reste la voie, le terrain sur lequel peut opérer la compréhension de l'espace insulaire. C'est d'ailleurs sur ce registre que s'exprime la mythologie ancienne et actuelle, sous des abords religieux puis littéraires. Rapprochement, analogie, métamorphose sont à l'œuvre. Images contre d'autres images, une mécanique fondée sur des perceptions sensorielles et des constructions mentales que recherche tout observateur, nourrissant par la suite des concepts et autres constructions rationnelles qui ont la tentation de verser dans l'onirisme. Comment rester insensible à l'abord de la côte et de sa plage de sable fin? On espère y trouver une vérité du monde, comme s'il y avait quelque chose à découvrir par là, comme si la réunion des contraires, des ambiguïtés donnait davantage de signification à la vie qui la traverse.

Tenons pour acquis que le trou et l'île ont en commun qu'ils sont des disjoncteurs du temps, de l'espace, comme cela a été dit plus haut. Pour le moins, il faut considérer qu'il y a une rupture du temps ordinaire, des habitudes, des manières d'habiter le monde et d'être au monde. En cela, obligeant à une rupture esthétique, l'île est une invitation, si l'on y consent, ou un enfer, si l'on est forcé. Dans tous les cas, ce déplacement sur l'échiquier du monde implique une perception autre à l'origine d'un disparaître que chacun recherche sans toujours le savoir.

Habiter est concomitant de l'apparaitre, intervenant juste après, lequel procède du même matériau et de la même technique de soi : le symbole et la symbolisation. Aller sur l'île c'est éprouver la contingence de la terre, éprouver un déterminisme géographique, autorisant la décompression du monde, une dissolution de son être. On recherche dans l'île l'exception face à la routine continentale. On souhaiterait que la pensée mythologique qui enchâsse l'espace insulaire devienne réelle dans un acte performatif sans précédent. L'île n'existe pas, n'existent que les conditions humaines qui la rendent possible dans une pensée faite action et pouvoir de transformation. Dire que l'île est une terre ou un espace entouré d'eau n'est pas suffisant, voire faible. C'est un fait acquis avant même que l'on écrive ces quelques mots.

Une île est déjà une terre d'élection avant même d'être choisie du fait de son caractère si particulier. C'est ce que pressent chaque arrivant au point d'imaginer que la vie insulaire se déploie de façon extraordinaire, pour le mieux, pour le pire, pour l'authenticité, loin de tout syndrome obsidional de celui qui vivrait l'insularité comme un enfermement.

Grâce à la clôture insulaire, l'île offre la possibilité au nouvel arrivant de disparaître, de se retirer. Vivre sur une île est un moyen de compenser un système routinier, de réguler une pression du monde car ce territoire est aux antipodes, une sorte d'antimonde, le lieu du relâchement et du lâcher-prise par excellence. Instinctivement chacun ressent sans être capable de le théoriser que disparaître reste l'acte suprême après l'apparaître, un acte éthique qui ne contredit pas l'habiter bien au contraire. Disparaître en est le prolongement, une variation dans une pleine réalisation de soi. 
Quand l'acte est volontaire, disparaître subsume l'humanité, à la condition d'entériner l'emploi du mode symbolique, au travers du corps et de sa posture, de la bouche et de ses mots. Car l'île agit comme une cellule de dégrisement, l'entrée dans le monde s'organise parfois dans sa fuite, sa sortie, dans une sorte de désenvoûtement. En cela, l'île constitue le trou d'air que certaines personnes recherchent afin de rééquilibrer, de relativiser les injonctions du monde à apparaître, qui plus est selon son bon vouloir. L'île est donc le lieu d'une épreuve de vérité qui n'a pas de prix. L'étendue d'eau protège et assure le processus, à la manière d'une prison dont les murs garantissent le procédé physico-chimique qui s'y déroule. Ici ajoutons le ressenti psychologique, voire le choc culturel avec l'éloignement.

C'est toute une grammaire de l'être qui diffère et qui pour le coup s'engramme dans une imagerie autre, allant jusqu'à transpirer dans des racines nouvelles. L'insularisme ne compte pas car il passe sous silence le processus. Il faudrait entendre l'insularité comme un phénomène en cours ou les conditions qui permettent le processus de dégrisement du monde, un processus dont la finalité serait de disparaître, posture éthique à la frontière d'un horizon esthétique peu banal.

9 À la suite de Moles, de Meistersheim, je reste persuadé que l'espace insulaire ne peut s'aborder que par l'imaginaire, les représentations mentales avec son jeu d'images venues de l'intérieur ou de l'extérieur de l'île. Quant aux concepts construits par des chercheurs, il faudrait dire leur implication ou leur participation personnelle qui alimentent ce même imaginaire. L'observation donne souvent sens à la situation vécue et étudiée.

Pour partie l'espace insulaire et le trou se superposent dans leur définition; se signalent parfois de la même façon, connaissant les mêmes difficultés à être définis. On comprend que l'on est en présence d'une surface sensible qui disjoncte la continuité, piégeant les hommes ou leur rendant d'immenses services. Si l'espace insulaire met à l'épreuve quelques lois humaines et celles de la république, il reste l'habiter et l'apparaître, ce qui fait que l'homme devient humain. Quand le trou fait trébucher, fait disparaître, l'île produit une autre forme de cristallisation géologique et subjective de l'être. L'espace insulaire invite à un disparaitre dans ce qu'il y a de plus fort, lequel serait un apparaître magnifié, augmenté et auréolé à la limite de la transcendance, loin de l'influence continentale, délestée du temps et de l'espace pour mieux le dépasser encore.

\section{BIBLIOGRAPHIE}

BONNEMAISON, Joël (1986). La dernière île. Paris : Arléa.

FOUCAULT, Michel (1984). Des espaces autres, Hétérotopies. Dits et Écrits. Paris : Gallimard.

FULIGNi, Bruno (1997). L'État, c'est moi. Histoire des monarchies privées, principautés de fantaisie et autres républiques pirates. Éd. de Paris-Max Chaleil. 
FUMEY, Gilles (2002). « Géographie des cryptarchies », Mappemonde, n66-2, pp.29-32.

HAINARD, Jacques et KAEHR, Roland (1990). Le trou. Neuchâtel : Musée d'ethnographie.

HeIDEgGER, Martin (1958). Bâtir Penser Habiter. Essais et conférences. Paris : Gallimard.

MEISTERSHEIM, Anne (1988). « Insularité, insularisme, iléité, quelques concepts opératoires », Cahiers de l'institut de développement des îles méditerranéennes, ${ }^{\circ} 1$, pp.96-120.

MEISTERSHEIM, Anne (2001). Figures de l'île. Ajaccio : DCL.

NoRA, Philippe (2003). « Du spirituel dans l'île », Tracés, n³, pp.9-23.

PÉRON, Françoise (1993). Des îles et des hommes, l'insularité aujourd'hui. Rennes : Ouest-France.

REBOUT, Lionel (2019). Peine perdue ! Cahiers de prison. Besançon : Éditions Phanères.

TAGLIONI, François (2006). « Les petits espaces insulaires face à la variabilité de leur insularité et de leur statut politique ", Annales de Géographies, n652, pp.664-687.

TRABELSI, Mustapha (2005). « Insularité et imaginaire », in Mustapha Trabelsi (éd.). L'insularité. Clermont-Ferrand : Presses Universitaires de Blaise Pascal, pp.5-8.

ViLATTE, Sylvie (1991). « L'insularité dans la pensée grecque », Annales Littéraires de l'Univ. de Besançon, pp.225-234.

\section{NOTES}

1. Il sera bien difficile de définir toutes les notions soulevées dans l'article, lequel mériterait de larges développements. Peu de chercheurs en sciences humaines s'intéressent au trou. Il existe le livre de HAINARD, Jacques et KAEHR, Roland (1990) édité par le musée d'ethnographie de Neuchâtel.

2. Le monde grec ancien connaît le trou sous bien des formes. Homère l'évoque au travers des grottes, du Cyclope Polyphème, entre autres figures.

3. En France, l'île de Ré, le château d'If, l'île Sainte-Marguerite. En Méditerranée, Goli Otok, Bastøy, Makronissos, l'île de Pianosa, l'île de Gorgone, Imarali . En Suède, Långholmen. Proche du continent américain, Alcatraz, Rikers Island, les îles du Salut, Guantanamo, l'île Coiba, l'île Dawson, l'île El Frontón, les îles Tres Marías, l'île Martín Garcia, l'île de la Jeunesse. Dans l'Océanie, l'île Nou, l'île Marie-Louise, Nusa Kambangan. Proche du continent asiatique, le bagne de Poulo Condor, l'île Christmas. Proche du continent africain, Robben Island, l'île de Changuu.

4. Voyez plutôt l'île Ratonneau, l'île aux Grues, Ellis Island, l'île aux Vainqueurs ; l'île Gruinard, l'île de Trébéron, l'île des Morts, les îles de Mainau et de Reichenau.

5. Voyez l'île du Lido San Michele, l'île Europa, l'île Lavezzi, Hart Island, l'île des Dékabristes, l'île Spiekeroog. Même le cimetière des chiens d'Asnières.

6. Par exemple, les îles Milliau, Gavrinis, de Carn, Renote, Guenioc, Hoedic.

7. Sur Arte, il y a eu une émission de 18 minutes intitulée Blow Up sur les îles dans le cinéma. Dans certains cas, l'île apporte un huis clos nécessaire à la narration et à son unité.

8. À l'île Maurice, on trouve le Trou-aux-Biches, Trou-aux-Cerfs et le Trou d'Eau Douce, le Trou Kanaka, non loin le Trou d'Argent sur Rodrigues ; en Haïti, le Trou-du-Nord (twou dino en créole) ; le Money Pit sur l'île Oak; le Trou de fer, le Trou blanc ou la cascade du Trou noir à La Réunion; en métropole à Groix, Trou de l'Enfer, Trou du Tonnerre.

9. Parmi celles-ci, voyez l'île d'Elleore, le territoire d'Enenkio, Forvik, les îles Lagoa, Minerva aux Tonga, Royaume de Tavolara.

10. Voyez l'île de Hans, l'île de Julia, objet de lutte diplomatique, la principauté d'Outer Baldonia dans les îles Tusket. 


\section{RÉSUMÉS}

Le trou et l'île sont liés en tant qu'ils sont une hétérotopie, autrement dit un interrupteur du temps et de l'espace. Au-delà de l'anecdote, nous montrons que le trou constitue et organise l'île de bien des façons. Comment le trou permet ou change l'insularité ? Il s'agira d'envisager le trou concrètement mais aussi en tant que représentation mentale. Il faudra peut-être dire les particularismes de ce couple si peu associé, entre ouverture et fermeture d'une société humaine, fondant une nouvelle subjectivité.

As heterotopia, the hole and the island are connected, because they are a switch of time and space. Beyond the anecdote, we show that the hole constitutes and organizes the island in many ways. How does the hole allow or change insularity? It will be a question of considering the hole concretely but also as a mental representation. We need perhaps to say the particularisms of this rarely associated couple, between opening and closing of human society, founding a new subjectivity.

\section{INDEX}

Mots-clés : hétérotopie, trou, représentation insulaire, subjectivité, esthétique

Keywords : heterotopia, hole, insular representation, subjectivity, esthetic

\section{AUTEUR}

\section{LIONEL REBOUT}

Université Paris Ouest Nanterre

19.prolix[at]gmail.com 